\title{
Memory attributions for choices: How beliefs shape our memories
}

\author{
Linda A. Henkel \\ Fairfield University, Ihenkel@fairfield.edu
}

M. Mather

Follow this and additional works at: https://digitalcommons.fairfield.edu/psychology-facultypubs Copyright 2007 Elsevier, Journal of Memory and Language NOTICE: this is the author's version of a work that was accepted for publication in Journal of Memory and Language. Changes resulting from the publishing process, such as peer review, editing, corrections, structural formatting, and other quality control mechanisms may not be reflected in this document. Changes may have been made to this work since it was submitted for publication. A definitive version was subsequently published in Journal of Memory and Language, [57, 2 (2007)] DOI: 10.1016/j.jml.2006.08.012

\section{Peer Reviewed}

\section{Repository Citation}

Henkel, Linda A. and Mather, M., "Memory attributions for choices: How beliefs shape our memories" (2007). Psychology Faculty Publications. 8.

https://digitalcommons.fairfield.edu/psychology-facultypubs/8

\section{Published Citation}

Henkel, L. A., \& Mather, M. (2007). Memory attributions for choices: How beliefs shape our memories. Journal of Memory and Language, 57.2, 163-176.

This item has been accepted for inclusion in DigitalCommons@Fairfield by an authorized administrator of DigitalCommons@Fairfield. It is brought to you by DigitalCommons@Fairfield with permission from the rightsholder(s) and is protected by copyright and/or related rights. You are free to use this item in any way that is permitted by the copyright and related rights legislation that applies to your use. For other uses, you need to obtain permission from the rights-holder(s) directly, unless additional rights are indicated by a Creative Commons license in the record and/or on the work itself. For more information, please contact digitalcommons@fairfield.edu. 
Running Head: MEMORY ATTRIBUTIONS FOR CHOICES

\author{
Memory Attributions for Choices: How Beliefs Shape Our Memories \\ Linda A. Henkel \\ Fairfield University \\ Mara Mather \\ University of California at Santa Cruz
}

Address correspondence to:

Linda Henkel

Department of Psychology

Fairfield University

Fairfield, CT 06824

203-254-4000x3269

lhenkel@mail.fairfield.edu 


\begin{abstract}
When remembering past choices, people tend to attribute positive features to chosen options and negative features to rejected options. The present experiments reveal the important role beliefs play in memory reconstruction of choices. In Experiment 1, participants who misremembered which option they chose favored their believed choice in their memory attributions more than their actual choice. In Experiment 2, we manipulated participants' beliefs by either "reminding" participants they chose an option they actually rejected or providing a correct reminder. Participants' memory attributions favored the option they believed they chose, both when that belief was correct and when it was erroneous. Furthermore, features attributed in a fashion favoring believed choices were more vividly remembered than features attributed in a non choice-supportive fashion. Thus beliefs at the time of retrieval about a choice lead to memory biases about both the valence and the vividness of remembered choice option features.
\end{abstract}

Key words: source memory; memory errors; memory for choices 


\section{Memory Attributions for Choices: How Beliefs Shape Our Memories}

Goals and beliefs at the time of memory retrieval often have a powerful influence on the way that memories are reconstructed. For example, convincing people to believe that frequent tooth brushing is harmful rather than beneficial leads them to recall brushing their teeth less often in the past few weeks (Ross, McFarland, \& Fletcher, 1981), and convincing people to believe that extroversion is superior or inferior to introversion affects how many extroverted behaviors they recall engaging in (Sanitioso, Kunda, \& Fong, 1990).

An important part of reconstructing events is attributing information to its appropriate source (Johnson, Hashtroudi, \& Lindsay, 1993). For instance, was it Paul or Sue who told that joke? Did I actually lock the door or only imagine doing it? Beliefs about the possible sources of what one remembers can affect the attributions made about those memories (e.g., Marsh, Cook, \& Hicks, 2006; Mather, Johnson, \& De Leonardis, 1999; Sherman \& Bessenoff, 1999; Spaniol \& Bayen, 2002). For instance, knowing that someone is Republican makes people more likely to attribute conservative-sounding statements to that person than to other potential sources (Mather et al., 1999). Beliefs about how positive or negative a particular source is can also influence source attributions. After people read a list of positive and negative behaviors engaged in by two men, those who learned that Geoff is a college professor, happy, married, and employed whereas Mark is a blue-collar employee, unhappy, divorced, and temporarily out of work were more likely later to attribute the positive behaviors on the list to Geoff and the negative behaviors to Mark (Cook, Marsh, \& Hicks, 2003). Wishful thinking can also play a role in memory attributions. Participants who read a series of predictions by psychics and learn that one psychic is more often right in her predictions than the other psychic tend to attribute predictions with desirable outcomes to the accurate psychic and predictions with undesirable 
outcomes to the inaccurate psychic (Gordon, Franklin, \& Beck, 2005). Furthermore, previous studies have shown that beliefs can influence source attributions even when the information about the sources is presented after initial encoding, and so the influence of beliefs extends beyond just directing attention when first learning information (Bayen, Nakamura, Dupuis, \& Yang, 2000; Cook et al., 2003; Hicks \& Cockman, 2003). Such work is consistent with the large body of studies showing that post-event information can impact the accuracy of what one remembers (e.g., Lane, Mather, Villa, \& Morita, 2001; Pizarro, Laney, Morris, \& Loftus, 2006; Zaragoza \& Mitchell, 1996).

The present studies seek to examine the extent to which beliefs can influence memory attributions about past choices. The objective of a choice generally is to pick the best option. Thus, after making a choice, you are likely to harbor the belief that the chosen option was better than the options you rejected. Motivation may also play a role in this process, as remembering the option that you chose as being the best option should help reduce regret about your choice. Indeed, several recent studies have shown that people tend to remember in ways that favor an option they had chosen over an option they had rejected. Specifically, when participants selected which of two options they would choose (e.g., two potential apartment rentals), they later showed a choice-supportive bias in their memory attributions (Mather \& Johnson, 2000; Mather, Shafir, \& Johnson, 2000, 2003). That is, they were more likely to attribute positive features (e.g., "sunny and bright") to the option they chose and attribute negative features (e.g., "small bedroom") to the option they rejected. This bias in favoring the chosen option in memory occurs not only when people make the choice themselves but also when a choice is made on their behalf that they are led to believe is in their best interest (Benney \& Henkel, 2006). However, people do not show choice-supportive biases when choices are made randomly for them (Benney \& 
Henkel, 2006; Mather et al., 2003).

Thus far research has provided important information about the circumstances under which choice-supportive biases in memory are more or less likely to occur. The findings from these studies showing that choice-supportive biases in memory occur when people make their choices purposefully but not when choices are made randomly suggest that the biases may be the result of people's belief that the option they chose is better. However, other factors may have led to the memory attributions favoring the chosen option over the nonchosen option, such as selective encoding due to information search processes biased in favor of a preferred alternative (Brownstein, 2003) or dissonance reduction immediately after the choice (Festinger, 1957). To better understand the role that beliefs play in memory attributions about past choices, the present studies examine instances when people have an incorrect belief about what their previous choice was. By placing beliefs about choices and the actual choice in opposition, we can isolate the effects of belief on memory for choices from other factors.

In addition, in the second experiment, we examined the role of beliefs on the vividness and qualitative characteristics of the memories. Although previous studies have examined how qualitative characteristics of false memories differ from characteristics of accurate memories (e.g., Arbuthnott, Geelen, \& Kealy, 2002; Bredart, Lampinen, \& Defeldre, 2003; Heaps \& Nash, 2001; Hoffman, Garcia-Palacios, Thomas, \& Schmidt, 2001; Karpel, Hoyer, \& Toglia, 2001; Kealy \& Arbuthnott, 2003; Mather, Henkel, \& Johnson, 1997; Neuschatz, Payne, Lampinen, \& Toglia, 2001; Norman \& Schacter, 1997), researchers have not investigated how beliefs about how desirable the memory is might influence its vividness. When remembering features from past choice options, positive features of chosen options and negative features of rejected options should be more satisfying to remember than non choice-supportive features. An interesting 
question is whether the desirability of a particular memory might influence how vivid it feels.

Participants in the present studies were given several descriptions of pairs of options

(e.g., two potential cars, two potential roommates) and asked to choose one of the two options within each pair. All options had both positive and negative features. Later, participants were asked to remember which features had been associated with a given option. Prior studies have found that participants sometimes misremember which of the two options they had originally chosen (e.g., Mather \& Johnson, 2000). To maximize the possibility of this occurring, participants in the present studies completed the feature attribution memory task 2 days after having made their choices in Experiment 1 and 1 week later in Experiment 2. If beliefs at the time of retrieval about the chosen option do indeed have an impact on memory attributions, then people's attributions should favor the option that they believe they chose - whether their belief is correct (they really did choose that option) or incorrect (they are misremembering which option they chose). Thus for both correctly remembered choices and incorrectly remembered choices, people should attribute relatively more positive features to the option they believe they chose, and more negative features to the option they believe they rejected.

\section{Experiment 1}

\section{Method}

Participants. Eighty undergraduates participated for partial course credit (65 women and 15 men). Ages ranged from 18 to 24 years old $(M=21.8, S D=6.6)$. Three additional participants did not return for the second session and two other participants did not indicate which options they had chosen. Data from these participants were not included in the analyses.

Materials. We used five choice scenarios that each included two options. These were choices between roommates, summer internships, apartments, cars, and potential dating partners 
(for an example, see the car choice in Table 1). There were 10-12 features listed for each of the two options in a scenario. Each option included both positive and negative features, the valence of which was established through prior norms. An equal number of positive features were randomly assigned to each of the two options in a scenario, and an equal number of negative features were as well. Thus within a given scenario, both options had the same number of positive features and the same number of negative features. For each scenario, there was a separate memory test consisting of a randomly ordered list of the items from each option intermixed with three to five positive and three to five negative new features.

Procedure. Participants were asked to read through each choice scenario at their own rate and select one of the two options by circling it. Instructions emphasized that they should take their time and carefully make their choice for each scenario. Each scenario was presented one at a time, and after participants made their choice, the next scenario was presented. The five scenarios were presented in a randomized order to each participant. They returned 2 days later to complete the surprise memory tests in which they were asked to indicate which option they had originally chosen and which option each feature had been associated with, or whether it was new. Thus for each feature, they were to chose from three alternatives: option A, option B, or new. The order of the memory tests was randomly determined for each participant.

Results

An alpha level of .05 was used for all statistical analyses and partial eta squared values $\left(\eta_{p}{ }^{2}\right)$ are reported to indicate effect sizes. All $t$ tests were two-tailed.

Memory accuracy. The proportion of studied items called old ("hits"), the proportion of new items called old ("false alarms"), and the proportion of hits correctly attributed to their original option are presented in Table 2 separately for scenarios with correctly remembered 
choices and misremembered choices. All participants correctly remembered at least some of their choices, and 50 participants were wrong about which option they chose for at least one choice. Of these 50, 32 incorrectly remembered one choice, 15 incorrectly remembered two choices, 1 incorrectly remembered three choices, 2 incorrectly remembered 4 choices, and 0 incorrectly remembered all 5 choices. The number of individuals who incorrectly remembered their choice for a given scenario ranged from $11(14 \%)$ to $21(26 \%)$.

Individual $t$ tests were conducted to compare memory performance for positive versus negative features for each of the 6 dimensions in Table 2. In general, participants were more accurate at remembering negative features than positive features (as in Mather \& Johnson, 2000; but see also Mather, Knight, \& McCaffrey, 2005, Experiment 3), and this was true for both old and new features (thus a "mirror effect' was shown, Glanzer \& Adams, 1985). Specifically, participants both recognized and identified the source of negative features more accurately than positive features (Rows 1, 3, and 4 of Table 2) and made fewer false alarms for negative than for positive features (Rows 2 and 5), with all $5 t$ tests yielding $p<.005$. The only exception to this pattern of better memory for negative features than for positive features was the nonsignificant difference in participants' source identification accuracy when they misremembered which option was chosen (Row 6), $t(49)<1$.

When comparing correctly remembered and misremembered choices for those 50 participants who had at least one of both, there were no significant differences in hit or false alarm rates (both $F<1$ ), but participants' source accuracy was significantly better when they correctly remembered which option they had chosen $(M=.63, S E=.01)$ than when they misremembered which option they had chosen $(M=.56, S E=.02), F(1,49)=9.30, M S E=.03$, $p<.01, \eta_{p}^{2}=.16$ 
Choice-supportive memory attributions. Of primary interest was the extent to which participants made attributions of positive and negative features to the chosen or rejected options. Source attributions were calculated in two different ways to assess this. The first set of analyses use a dependent measure typically used in many studies of source monitoring (see Henkel \& Franklin, 1998). This is based on the proportion of studied features correctly attributed to the chosen or rejected option, given that the features were correctly recognized as old. The second set of analyses use a dependent measure known as an asymmetry score that was developed specifically to examine choice-supportive memory attributions. Asymmetry scores were calculated using the method outlined by Mather et al. (2000) in order to reveal whether participants' memory attributions favored their chosen options or not (i.e., whether they assigned relatively more positive features to the chosen option and more negative features to the rejected option). ${ }^{1}$ This particular measure is useful because it provides a composite score indicating the extent to which participants made attributions favoring the chosen or nonchosen option, thus allowing direct comparisons across conditions of the degree of choice-supportiveness. In addition, this measure controls for spurious effects that may be due to unequal numbers of participants having selected one option over another when making their initial choice. ${ }^{2}$ Positive scores indicate attributions favoring the chosen option, and negative scores indicate attributions favoring the nonchosen option, with scores of zero showing a bias toward neither option.

Figure 1 presents the proportion of features attributed to the option remembered as chosen or rejected as a function of feature valence. The top left panel shows source attribution accuracy rates when participants correctly remembered which option was chosen, and the top right panel shows attributions when participants incorrectly remembered which option was chosen. A 2 (feature valence: positive, negative) x 2 (option attributed to: remembered as 
chosen, remembered as rejected) repeated-measures ANOVA was conducted for the scenarios in which participants correctly remembered which option they chose, and a significant feature valence $\mathrm{x}$ option-attributed-to interaction was found, $F(1,79)=25.74, p<.001, \eta_{p}{ }^{2}=.25$. As predicted, participants attributed more positive features to the chosen option than to the rejected option, $t(79)=6.32, p<.001$. They attributed slightly though not significantly more negative features to the rejected option than to the chosen option, $t(79)=1.62, p=.10$.

A separate $2 \times 2$ ANOVA was also conducted for the cases in which participants incorrectly remembered which option they chose, and again, a significant feature valence $\mathrm{x}$ option-attributed-to interaction was found, $F(1,49)=103.71, p<.002, \eta_{p}^{2}=.18$. Here, participants attributed significantly more positive features to the option they believed they chose than to the option they actually chose, $t(49)=29.92, p<.005$, and they attributed more negative features to the option they believed they rejected than to the option they actually rejected, $t(49)=$ $2.27, p<.03$.

Separate analyses were conducted for correctly remembered choices and for incorrectly remembered choices because not all participants incorrectly remembered a choice. Direct comparisons of attributions for correctly remembered choices and incorrectly remembered choices can be made only for the 50 participants who incorrectly remembered at least one of their choices. The direct comparison also revealed a significant feature valence $\mathrm{x}$ optionattributed-to interaction, $F(1,49)=17.08, p<.001, \eta_{p}{ }^{2}=.26$, but no significant interaction between choice accuracy, feature valence, and option-attributed-to, $F(1,49)=1.43, p=.24$. Thus participants' attributions were choice-supportive both for options that they correctly remembered and for options that they incorrectly remembered.

Choice-supportive attribution biases can also be examined for new items erroneously 
claimed as belonging to the chosen or rejected option. The proportion of positive and negative new items attributed to the option remembered as chosen or rejected was therefore examined both for scenarios in which participants correctly remembered their choices and for scenarios in which they incorrectly remembered their choices. These data are presented in the bottom panel of Figure 1. As in the analyses for studied items, a 2 × 2 ANOVA indicated a significant feature valence $\mathrm{x}$ option-attributed-to interaction for correctly remembered choices, $F(1,79)=18.42$, $\mathrm{p}$ $<.001, \eta_{p}^{2}=.19$. When participants correctly remembered which option was chosen, they attributed more positive features to the chosen option than to the rejected option, $t(79)=2.40, p$ $<.01$, and more negative features to the rejected option than to the chosen option, $t(79)=4.00, p$ $<.001$. Likewise, a significant feature valence $\mathrm{x}$ option-attributed-to interaction was found for incorrectly remembered choices, $F(1,49)=7.14, p<.01, \eta_{p}{ }^{2}=.13$. When participants misremembered which option was chosen, they attributed more positive features to the option they believed they chose than to the option they believed they rejected, $t(49)=2.51, p<.01$, and slightly though not significantly more negative features to the option they believed they rejected than to option they believed they chose, $t(49)=1.51, p=.14$. An additional analysis using only the subset of participants who misremembered at least one of their choices and correctly remembered the others showed a significant valence $\mathrm{x}$ option-attributed-to interaction as well, $F(1,49)=17.39, p<.001, \eta_{p}{ }^{2}=.26$, with no significant interaction between choice accuracy, feature valence, and option-attributed-to $F(1,49)=0.14, p=.71$. Thus participants' attributions of new items were choice-supportive both for options that they correctly remembered and for options that they incorrectly remembered.

Analyses were also conducted based on asymmetry scores for both correctly and incorrectly remembered choices, and the results of these analyses are consistent with the results 
of analyses based on conditionalized proportions. For the choices in which participants correctly remembered which option they had selected, asymmetry scores were significantly greater than zero, $M=.28, \mathrm{SE}=.06, t(79)=4.63, p<.001$, and thus revealed a bias favoring chosen options. For the choices in which participants incorrectly remembered which option they chose, participants significantly favored the options they thought they chose, rather than the ones they actually chose (with positive scores indicating a bias in favor of remembered choices and against the actual choices), $M=.56, \mathrm{SE}=.15, t(49)=3.80, p<.001$. Among the 50 participants who misremembered which option they had chosen for at least one scenario and correctly remembered which option they had chosen for other scenarios, there was no significant difference in how choice supportive they were for the correctly $(M=.28, S E=.09)$ and incorrectly remembered choices $(M=.56, S E=.15), t(49)=1.67, p=.10$. Shown in the top panel of Table 3 are the mean choice-supportive asymmetry scores for these 50 participants separately for correct and incorrect attributions of old features as well as for incorrect attributions of new features. A 2 (Belief about which option was chosen: Correct, Incorrect) x 3 (Attribution type: Correct Old Feature, Incorrect Old Feature, Incorrect New Feature) ANOVA revealed no significant effects (all ps $>.10$ ). Thus, participants had similar choice-supportive biases in their attributions for both old and new features.

\section{Discussion}

The present study assessed the impact of belief about choices on people's attribution of positive and negative features to the options they believe they chose and the options they believe they rejected. To create a situation where people would misremember some of their choices, a 2day delay between having made the choices and assessment of memory was used. Recognition accuracy was reasonably high (the average hit rate was about $86 \%$ ), even with this long retention 
interval, and did not vary whether participants correctly or incorrectly remembered the chosen option. Overall source identification accuracy was modest, especially when participants misremembered which option was chosen. However, the absolute rates of source accuracy are a function of the very phenomenon under investigation. That is, when participants misremember an option, we argue that their source attributions will be a function of which option they believe they chose rather than which option they actually chose.

The findings from Experiment 1 indicate that people's memory attributions favor the option they believe they chose over the option they believe they rejected, even when they incorrectly remember which option was in fact chosen. This illustrates the strong impact belief at the time of retrieval can have on one's memory. Whereas prior studies have shown that beliefs are an important component of reconstructive memory processes in general (e.g., Cook et al., 2003; Mather \& Johnson, 2003; Mather et al., 1999), the role that beliefs play in memory attributions about past choices has not been addressed and was thus the primary focus of the present study.

The fact that choice-supportive biases favoring the option believed to have been chosen over the option believed to have been rejected were found not only for features that were part of the original scenarios but also for new features that were not presented at all is important to note because it indicates that the bias cannot be accounted for solely by selective encoding. While deciding which option to choose, participants may simply pay more attention to the positive features that draw them to the option they wind up choosing or to the negative features that lead them to reject the other option. However, if this were the sole factor driving the later memory attributions, then attributions should be choice supportive for originally studied features but not for new features. 
The two different measures of source attributions used here allow further confidence in the conclusion that one's beliefs about a choice can influence what one remembers about the chosen and rejected options. Because the proportion measure that was used was conditionalized on correct recognition, the choice-supportive patterns obtained cannot simply be the byproduct of differential hit rates. Furthermore, although people tended to better remember negative rather than positive features overall (both in terms of their hit rates and their source attributions), they nonetheless showed different patterns of attributions for negative features depending on what option they believe they chose, and different patterns of attributions for negative over positive features depending on their beliefs about what option was chosen. In addition, the analyses using asymmetry scores also yielded patterns of choice-supportive attributions, based on a dependent measure that is not affected by spurious effects that may arise due to unequal numbers of participants selecting one option over the other in a given scenario. Taken together, these findings support the argument that beliefs - both correct and incorrect - about which option was chosen give rise to memory attributions that favor the option remembered as chosen over the option remembered as rejected.

However, because participant's beliefs about which options were chosen and which options were rejected were not directly manipulated, the results from the present study do not rule out the possibility that differences in the features best remembered can contribute to the bias shown. It may be that a choice-supportive bias in memory attributions is not directly the result of a belief about choice per se. Instead, people's belief about which option they chose and the choice-supportive attributions they make may both be the outcome of which features are remembered best. Specifically, if negative features from Option A are recalled most vividly, participants may both tend to believe they rejected that option (and chose the other option) and to 
attribute positive features to the option they believe they chose and negative features to the option they believe they rejected. If this were the case, beliefs about the choice itself would not play a critical role. Experiment 2 was thus designed to better understand the way in which beliefs about past choices shape one's memories and attributions made about those memories.

\section{Experiment 2}

In Experiment 2, we misled participants about which option they had chosen for some choices to more stringently test the role of belief about choices in choice-supportive memory biases. We gave participants a series of choices and then brought them back for memory tests 1 week later. At that point, we "reminded" them of which option they had chosen for each choice. For each participant, we misinformed them about two choices and correctly informed them about two choices. If the belief that whichever option one chose was the better option is the critical factor underlying choice-supportive memory, choice-supportive biases should be just as strong in the misleading-reminder condition as in the correct-reminder condition.

This study also provided us with the opportunity to examine how beliefs about sources might influence the qualitative characteristics of the memories attributed to those sources. In particular, we were interested in whether features attributed in a belief-consistent manner would seem more vivid than features that do not fit as well with one's expectations. Previous studies examining the qualitative characteristics of memories have typically focused on how the characteristics differ depending on the source or nature of the memory. For example, participants who hear a list of words that are all semantically related to a lure word and later incorrectly remember hearing the lure word give lower ratings of perceptual detail for the lure words than for the correctly remembered words (Mather et al., 1997; Norman \& Schacter, 1997). Along the same lines, previous studies have found that, in comparison to neutral memories, 
emotional memories tend to be remembered more vividly and be given higher ratings for their qualitative characteristics (Schaefer \& Philippot, 2005; Talarico, LaBar, \& Rubin, 2004).

However, previous research has not examined how beliefs at the time of retrieval about the source of the attributed information might affect how vividly it is remembered.

By manipulating participants' beliefs about the sources at retrieval, in this study we evaluated whether the subjective qualities of memory are also subject to the same reconstructive processes as other aspects of episodic memory.

\section{Method}

Participants. Sixty-one undergraduates (46 women, 15 men) participated for course credit. Ages ranged from 17 to $21(M=18.7, S D=1.0)$. An additional 6 participants did not return for the second session, and 1 participant did not follow instructions on the memory test and so that person's results were not included in the analyses.

Materials and procedure. The materials and procedure were identical to Experiment 1 except that participants returned 1 week later rather than 2 days later for the second session, and on the memory tests, we asked participants not only to indicate which option each feature had been associated with, or whether it was new, but we also asked them to rate their confidence that the feature was associated with the option they attributed it to. In addition, we asked them to rate: (a) how clearly they remembered their feelings and emotional reactions about the feature when they first made the choice; (b) how clearly they remembered any other non-emotional thoughts about this feature or associations to it when they first made the choice; and (c) overall, how vividly and clearly they remembered the feature or details about it. Each of these ratings was made on a 1-5 scale ranging from not at all clear to very clear. Participants were asked not to give these ratings for features they said were new. 
At the top of the memory questionnaire for each choice scenario, we listed the two choice options, with one of them circled ahead of time by the experimenter. We told participants that the option they selected last week was the one that was circled. The first scenario tested was always the car choice, and the option participants had actually chosen was correctly circled for this one. This served as a filler scenario to help establish the legitimacy of our reminders and was not included in the data analyses. Participants were given correct reminders about the choices they had made for two of the other four scenarios and misinformed about the other two scenarios. Which two scenarios had misleading reminders were counterbalanced across participants and the four scenarios were presented in random order.

A final questionnaire served as a manipulation check for the deception about the chosen options. For each scenario, the same option was circled as on the source attribution test and participants were instructed to list two reasons they chose it, if they could remember. In addition, for each scenario, they rated how well they remembered choosing that option (where 1 $=$ do not remember $\ldots 5$ = very clearly remember .

Results

Manipulation checks. On the final questionnaire, 4 participants said they thought they had chosen a different option for at least one of the misleading-reminder scenarios. In addition, another 9 participants left the "reasons why I picked this option" question blank for at least one of the misleading-reminder options. As most (7) of these participants left the reasons fields blank for all five scenarios, this was probably an indication of forgetting or laziness rather than disbelief about having chosen a particular option. However, to be conservative, in the subsequent analyses we only included the 48 participants who provided us with reasons why they had chosen each of the misleading-reminder options and who did not express any doubts about 
having chosen those options.

When asked how clearly they remembered choosing each option, the average rating was between a 3 ("somewhat remember choosing that [car/roommate, etc.]") and a 4 ("fairly clearly remember choosing that [car/roommate, etc.]") on the 5-point scale, with no significant difference between ratings for the two misleading-reminder choices $(M=3.34, S E=.11)$ and the two correct-reminder choices $(M=3.52, S E=.12), t(47)=1.53, p=.13$. Thus, we appear to have been successful at convincing these participants that they chose the options we indicated they had chosen.

Memory accuracy. Hits, false alarms, and source attribution scores were calculated the same way as in Experiment 1 (see Table 4 for means). As in Experiment 1, memory tended to be better for negative features than positive features, but the difference was not as pronounced, with significant effects only seen for false alarms in the correct-reminder condition, $t(47)=2.02, p$ $<.05$, and the misleading-reminder condition, $t(47)=3.25, p<.01$ (Rows 2 and 4 in Table 5).

Comparisons of the correct-reminder and misleading-reminder conditions showed similar hit rates for the two conditions for both positive and negative features (all $t \mathrm{~s} \leq 1$ ), but source identification accuracy was higher in the correct-reminder condition than the incorrect-reminder condition for both positive features, $t(47)=4.12, p<.001$, and negative features, $t(47)=4.16, p$ $<.001$. Thus, misleading participants about which option they had chosen did not affect their recognition accuracy, but did impair their source accuracy.

Choice-supportive memory attributions. Attributions of positive and negative features to the chosen or rejected options were examined in the same manner as in Experiment 1 . The top panel of Figure 2 presents the proportion of features attributed to the option remembered as chosen or rejected as a function of feature valence and type of reminder about the chosen option. 
A 2 (feature valence: positive, negative) x 2 (option attributed to: told was chosen, told was rejected) x 2 (reminder: correct, misleading) repeated-measures ANOVA was conducted, and a significant feature valence $\mathrm{x}$ option-attributed-to interaction was found, $F(1,47)=38.98, p<$ $.001, \eta_{p}{ }^{2}=.45$, without a significant three-way interaction, $F(1,47)=0.81, p=.37$. As predicted, when participants were given a correct reminder about their choice, they attributed more positive features to the chosen option than to the rejected option, $t(47)=7.44, p<.001$, though they did not attribute more negative features to the rejected option than to the chosen option, $t(47)<1$. When participants were incorrectly informed as to which option they had chosen, they attributed significantly more positive features to the option they were led to believe they chose than to the option they actually chose, $t(47)=7.44, p<.001$, though they attributed only slightly but not significantly more negative features to the option they believe they rejected than to the option they actually rejected, $t(47)=1.63, p=.11$.

False alarms to new items were also examined to determine whether people made attributions of new items in a manner that favored the chosen over the rejected option. These data are presented in the bottom panel of Figure 2. As in the analyses for studied items, a $2 \times 2 \times$ 2 ANOVA showed a significant feature valence $\mathrm{x}$ option-attributed-to interaction, $F(1,47)=$ 23.01, $p<.001, \eta_{p}{ }^{2}=.33$, and no significant three-way interaction $F(1,47)=0.02, p=.96$. When participants were given correct reminders as to which option was chosen, they attributed more positive features to the chosen option than to the rejected option, $t(47)=2.44, \mathrm{p}<.02$, and more negative features to the rejected option than to the chosen option, $t(47)=2.61, p<.01$. Likewise, when participants were given incorrect reminders as to which option was chosen, they attributed more positive features to the option they believed they chose than to the option they believed they rejected, $t(47)=2.88, p<.01$, and more negative features to the option they 
believed they rejected than to option they believed they chose, $t(47)=2.61, p<.01$.

Asymmetry scores were computed using the same method as in Experiment 1 to further examine whether participants' memory attributions favored the apparently chosen options (those circled by us) or not. Participants' overall asymmetry scores were significantly greater than zero and thus revealed a bias favoring apparently-chosen options for both the correct-reminder scenarios $(M=.47, S E=.08, t[47]=5.56, p<.001)$ and the misleading-reminder scenarios $(M=$ $.50, S E=.11, t[47]=4.62, p<.001)$. Indeed, there was no significant difference between the conditions, $t(47)=.28$, indicating that participants were just as choice-supportive for options they had actually rejected but that we told them they had chosen as they were for the options for which our reminders were accurate. A follow-up analysis computing asymmetry scores separately for correctly attributed old features, misattributed old features, and misattributed new features revealed significant choice-supportive asymmetry scores for each of these types of attributions, with no significant differences by type of attribution or condition (see the lower panel of Table 3 for means; all $p>.5$ ).

Memorial features. For analysis of the various subjective memorial characteristics (emotions, other thoughts, overall vividness), we coded each attribution of a feature to an option as choice-supportive or not choice-supportive, depending on whether the feature was positive or negative and the option was chosen or rejected (based on the options we told participants they had chosen, rather than on their actual choices). Separate 2 (attribution: choice-supportive or not) x 2 (reminder: correct or misleading) x 2 (feature valence: positive, negative) ANOVAs for each characteristic revealed that for each type of subjective rating, features attributed in a choicesupportive fashion (positive features attributed to apparently chosen options and negative features to apparently rejected options) were rated more highly than features attributed in a non 
choice-supportive fashion $\left(p<.05\right.$ for all ratings; $\eta_{p}{ }^{2}=.08, .17, .19, .21$ for confidence, emotions, thoughts, and vividness, respectively; see Figure 3). There were no significant effects of the type of reminder or significant interactions of type of reminder and attribution. Thus, participants rated the features they attributed in a choice-supportive fashion as being more vividly remembered, even when the "chosen option" was actually the option they had rejected.

An interesting question is whether this inflation of the memorial characteristics of features attributed in a choice-supportive way occurred for the new features. Not everyone falsely attributed new features to both chosen and rejected options, but among the 45 participants who did, new features attributed in a choice-supportive way were given higher average qualitative characteristic ratings $(M=3.02, S E=.10)$ than new features attributed in a non choice-supportive way $(M=2.77, S E=.13), t(44)=2.05, p<.05$. This finding that a vividness inflation effect occurred for new features never seen before indicates that biases at the time of retrieval must help create the effect.

\section{Discussion}

These findings further indicate that belief influences memory attributions for past choice options. When participants are lead to believe that they chose a particular option, they remember relatively more positive attributes having been associated with the chosen option and more negative attributes with the rejected option. These findings thus conceptually replicate and extend those from Experiment 1. In Experiment 1, participants' beliefs about their choices were examined by comparing memory attributions when they correctly remembered which option they had chosen to their attributions when they misremembered which option they had chosen. By directly manipulating people's beliefs about their choices by providing them with correct or misleading reminders as to which option they had originally chosen in Experiment 2, we were 
able to isolate the choice-supportive memory attribution biases seen to the beliefs held about their choice at retrieval. Differences in memorability of features cannot alone account for these findings.

The fact that relatively few participants questioned the misleading information they were given when they were told which of the options they had selected 1 week prior extends recent findings of "choice blindness" (Johansson, Hall, Sikstrom, \& Olsson, 2005). Participants asked to indicate which of two faces was most attractive and then handed one of the pictures and asked to describe their reasons for their choice rarely noticed when the experimenter surreptitiously swapped the pictures. The participants gave reasons for choosing the face they had not chosen, just as our participants gave reasons for the choice we said they had made. Furthermore, in our study, when asked how clearly they remembered choosing each option, the average rating was between "somewhat" and "fairly clearly" remember choosing that option, and the subjective vividness of the memory for the choice was not significantly greater when they were asked about the choice they had really made than when they were misled about which choice they had made. Thus people's subjective experience was that they were in fact remembering their choice from Session 1, even when they were misremembering it.

It is important to put these findings in the context of overall rates of memory performance. Even with the 1-week retention interval used in the present experiment, people's overall recognition of the attributes was reasonably good $(\sim 80 \%)$. Thus it is not simply the case that people have little memory for the choices and their associated features and are simply guessing on the memory attribution test. The fact that they show similar patterns of source attributions for old items as well as for new items indicates that their attributions are guided by their belief as to which option was chosen. Although overall source attribution rates were low, 
this may be precisely the circumstance under which people are more likely to rely on beliefs about their chosen option to reconstruct their memory for the associated features. These reconstructive processes no doubt are a combination of actual memory experiences as well as processes such as guessing and reasoning (this issue is discussed further in the general discussion).

Related to this point is the finding of greater source monitoring accuracy for scenarios in which correct reminders about choices were given than when misleading reminders were given, which is congruent with the finding in Experiment 1 of greater source monitoring accuracy when people correctly remember which option they chose than when they misremember. This finding suggests that beliefs help support accurate source attributions as well as influence inaccurate attributions. For instance, having made a choice between two cars, people may correctly remember that "the option I chose had the comfortable seats." If their belief about which option they chose is correct, they will have higher source attribution accuracy than if their belief about which option they chose is incorrect.

By deceiving participants at retrieval about which option they had chosen, we were also able to examine the effects of beliefs about sources on the qualitative characteristics of the features attributed to them. For the confidence rating, we asked participants how confident they were that the feature had been associated with that option. But the other ratings of qualitative characteristics were focused on the feature itself rather than on source memory (e.g., "How vividly and clearly do you remember this feature or details about it?") Results indicated that participants were not only more confident about the source of features attributed in a choicesupportive way, but also felt they remembered them more clearly than the features attributed in a non choice-supportive way. This vividness inflation occurred both when they were misled about 
which option they had chosen and when they were provided with correct reminders. In addition, the vividness inflation also occurred for the new features that were falsely attributed to one of the two options. Thus, this study suggests that features that support one's beliefs in terms of what they are associated with feel more vivid at the time of retrieval.

\section{General Discussion}

When remembering the features of past choice options, people have a choice-supportive bias in the way that they attribute these features (Mather \& Johnson, 2000; Mather et al., 2000, 2003). Previous studies reveal circumstances under which choice-supportive biases are more likely to be shown. For instance, younger adults show more choice-supportive biases when induced to think about their emotions about the choice (Mather \& Johnson, 2000). People do not show choice-supportive biases when a computer randomly selects the chosen option (Benney \& Henkel, 2006).

This latter research is consistent with the idea that beliefs about the chosen option may play a critical role, because choice-supportive biases are found when people are likely to believe that the option chosen was superior but not when they are unlikely to have that belief (e.g., when the choice is randomly made). However, the cause of the bias was not clear from these previous studies, as the process of making a choice provides many opportunities for bias (e.g., Mather et al., 2005). Selective attention at the time of encoding may make choice-supportive features more memorable later (Brownstein, 2003; Jonas, Schulz-Hardt, Frey, \& Thelen, 2001). Additionally, faced with cognitive dissonance about the positive features of the rejected option and the negative features of the chosen option, people may distort their memories immediately after making a choice in order to resolve their psychological discomfort (Elliot \& Devine, 1994; Festinger, 1957). The present studies investigate whether the belief at the time of retrieval that 
one chose Roommate A and rejected Roommate B is in itself enough to create a choicesupportive memory bias, even if which roommate is believed to have been the chosen one is erroneously remembered by the person or is randomly determined by the experimenter.

Our results support the notion that beliefs at the time of retrieval are sufficient to create choice-supportive memory. In Experiment 1 we examined instances in which people spontaneously misremember which of the options they chose. In these cases, people showed memory biases favoring their believed choices rather than their actual choices. In Experiment 2, we manipulated people's beliefs by misinforming them about which option they selected earlier. Participants had choice-supportive biases in memory for whichever option we told them they had chosen, whether or not they had actually chosen it. In addition, we found that when feature attributions favored the believed choice, those features were rated as being remembered more vividly with more associated phenomenal characteristics than when the feature attributions favored the other option. This finding that beliefs about what option was chosen and what option was rejected plays a critical role in memory reconstruction adds to our current understanding of choice-supportive memory bias by providing insights into a critical cause of the phenomenon.

One question we have received in response to these findings is how much they reflect “real” memory processes versus nonmemorial judgment processes involving guessing or reasoning. From the perspective of the source-monitoring framework, this is a false dichotomy, as memory is inextricably intertwined with judgment processes (Johnson et al., 1993). In order to interpret a currently activated mental representation (e.g., the statement "high mileage on odometer") as corresponding with a past event rather than just a new perception or thought, heuristic and systematic judgment processes must indicate that the qualitative features of the mental activation meet the criteria for a match with some source, such as one of the cars 
previously read about. Thus a currently activated mental representation is experienced as "a memory," even when it is based on misinformation (here, about the option that was chosen). Findings from Experiment 2 are consistent with this view. Very few participants caught onto the deceptive nature of the reminders we provided about their choices, and almost all were willing to provide reasons why they made their choice (see also Johansson et al., 2005). They reported moderate to high levels of confidence and vividness for the choices that they misremembered as well as for the choices they correctly remembered. Thus their subjective experience was that they were remembering in both cases. The present findings as well as numerous other studies attest to the fact that people's subjective experience of remembering can be quite erroneous (e.g., when people claim to "remember" having heard words on a list that were never presented, Mather et al., 1997; Norman \& Schacter, 1997; Roediger \& McDermott, 1995). Furthermore, overall recognition rates illustrate that people's general sense for what did and did not occur in the present study was reasonably intact. While source attribution accuracy rates were modest at best in the current studies, it is likely the case that that is precisely the circumstance under which beliefs are more likely to guide memory reconstruction, as is found in studies examining situations in which people are more likely to rely on schemas to guide their remembering (e.g., Mather \& Johnson, 2003; Mather et al., 1999; Spaniol \& Bayen, 2002). Future research can more closely examine whether decisions that are more meaningful and important are less likely to be impacted by beliefs and reconstructive memory biases, though certainly studies have suggested that even in many complex and meaningful everyday experiences, biases in memory can occur, such as when people remember their high school and test grades as higher then they actually were (Bahrick, Hall, \& Berger, 1996), women remember their pregnancy and childbirth experiences as more positive than they had documented at the time in their journals (Smith, 
1994), and older adults remember their past health and well-being as better than they originally reported (Kennedy, Mather, \& Carstensen, 2004).

According to the source monitoring framework, both the qualitative features of activated information and general knowledge influence source judgments (Johnson et al., 1993). Our findings not only demonstrate that beliefs about which option was chosen play a significant role in reconstructing memories of past choices, but that beliefs can influence the qualitative characteristics that comprise the subjective experience of remembering as well. This finding that manipulating people's beliefs influences the vividness of their memories has both practical and theoretical implications. Consider, for example, this finding in the context of social persuasion: Tactics that manipulate a consumer's beliefs about whether they previously chose an option can potentially alter the person's recollection and the vividness of the features comprising their memory. From a theoretical standpoint, models of memory must consider the complex interplay between mental representation and beliefs. Previous studies have examined qualitative characteristics to shed light on how various types of memories differ and how the nature of the qualitative features help determine source attributions (e.g., Comblain, D'Argembeau, \& Van der Linden, 2005; Hashtroudi, Johnson, \& Chrosniak, 1990; Johnson, Foley, Suengas, \& Raye, 1988; Mather et al., 1997; Norman \& Schacter, 1997; Schaefer \& Philippot, 2005; Talarico et al., 2004). The present findings indicate that the qualitative features of memories not only serve as input for source judgment processes, but that they are also likely to be influenced by one's beliefs at the time of retrieval. The interactive nature of the memorial characteristics and general knowledge about the situation may help make belief-consistent memory distortion seem more vivid and realistic than belief-inconsistent errors in source monitoring.

In conclusion, the present studies demonstrate the important role that beliefs play in 
reconstructing memories about past choices. The malleability of beliefs and memory attributions based on those beliefs observed here, although troubling perhaps in some regards as to how erroneous people's memories can be, are likely the byproducts of a cognitive system that generally is efficient at compensating for memories that become impoverished over time. In addition, it may well be the case that by having a memory system that is guided at times by our current beliefs, people are able to maintain a greater sense of satisfaction and well being with the choices they have made in their lives. 


\section{References}

Arbuthnott, K. D., Geelen, C. B., \& Kealy, L. L. K. (2002). Phenomenal characteristics of guided imagery, natural imagery, and autobiographical memories. Memory \& Cognition, 30, 519-528.

Bahrick, H. P., Hall, L. K., \& Berger, S. A. (1996). Accuracy and distortion in memory for high school grades. Psychological Science, 7, 265-271.

Bayen, U. J., Nakamura, G. V., Dupuis, S. E., \& Yang, C. L. (2000). The use of schematic knowledge about sources in source monitoring. Memory \& Cognition, 28, 480-500.

Benney, K. E., \& Henkel, L. A. (2006). The role of free choice in memory biases for past decisions. Manuscript submitted for publication.

Bredart, S., Lampinen, J. M., \& Defeldre, A. C. (2003). Phenomenal characteristics of cryptomnesia. Memory, 11, 1-11.

Brownstein, A. L. (2003). Biased predecision processing. Psychological Bulletin, 129, 545-568.

Comblain, C., D'Argembeau, A., \& Van der Linden, M. (2005). Phenomenal characteristics of autobiographical memories for emotional and neutral events in older and younger adults. Experimental Aging Research, 31, 173-189.

Cook, G. I., Marsh, R. L., \& Hicks, J. L. (2003). Halo and devil effects demonstrate valencedbased influences on source-monitoring decisions. Consciousness and Cognition, 12, 257278.

Elliot, A. J., \& Devine, P. G. (1994). On the motivational nature of cognitive dissonance: Dissonance as psychological discomfort. Journal of Personality and Social Psychology, $67,382-394$. 
Festinger, L. A. (1957). A theory of cognitive dissonance. Stanford, CA: Stanford University Press.

Glanzer, M., \& Adams, J. K. (1985). The mirror effect in recognition memory. Memory \& Cognition, 13, 8-20.

Gordon, R., Franklin, N., \& Beck, J. (2005). Wishful thinking and source monitoring. Memory \& Cognition, 33, 418-429.

Hashtroudi, S., Johnson, M. K., \& Chrosniak, L. D. (1990). Aging and qualitative characteristics of memories for perceived and imagined complex events. Psychology and Aging, 5, 119126.

Heaps, C. M., \& Nash, M. (2001). Comparing recollective experience in true and false autobiographical memories. Journal of Experimental Psychology: Learning, Memory, and Cognition, 27, 920-930.

Henkel, L. A., \& Franklin, N. (1998). Some comments on "Measuring memory for source: Some theoretical assumptions and technical limitations. Memory \& Cognition, 26, 678-680.

Hicks, J. L., \& Cockman, D. W. (2003). The effect of general knowledge on source memory and decision processes. Journal of Memory and Language, 48, 489-501.

Hoffman, H. G., Garcia-Palacios, A., Thomas, A. K., \& Schmidt, A. (2001). Virtual reality monitoring: Phenomenal characteristics of real, virtual, and false memories. Cyberpsychology \& Behavior, 4, 565-572.

Johansson, P., Hall, L., Sikstrom, S., \& Olsson, A. (2005). Failure to detect mismatches between intention and outcome in a simple decision task. Science, 310, 116-119. 
Johnson, M. K., Foley, M. A., Suengas, A. G., \& Raye, C. L. (1988). Phenomenal characteristics of memories for perceived and imagined autobiographical events. Journal of Experimental Psychology: General, 117, 371-376.

Johnson, M. K., Hashtroudi, S., \& Lindsay, D. S. (1993). Source monitoring. Psychological Bulletin, 114, 3-28.

Jonas, E., Schulz-Hardt, S., Frey, D., \& Thelen, N. (2001). Confirmation bias in sequential information search after preliminary decisions: An expansion of dissonance theoretical research on selective exposure to information. Journal of Personality and Social Psychology, 80, 557-571.

Karpel, M. E., Hoyer, W. J., \& Toglia, M. P. (2001). Accuracy and qualities of real and suggested memories: Nonspecific age differences. Journals of Gerontology Series B: Psychological Sciences and Social Sciences, 56, P103-P110.

Kealy, K. L. K., \& Arbuthnott, K. D. (2003). Phenomenal characteristics of co-created guided imagery and autobiographical memories. Applied Cognitive Psychology, 17, 801-818.

Kennedy, Q., Mather, M., \& Carstensen, L. L. (2004). The role of motivation in the age-related positivity effect in autobiographical memory. Psychological Science, 15, 208-214.

Lane, S., Mather, M., Villa, D., \& Morita, S. (2001). How events are reviewed matters: Effects of varied focus on eyewitness suggestibility. Memory \& Cognition, 29, 940-947.

Marsh, R. L., Cook, G. I., \& Hicks, J. L. (2006). Gender and orientation stereotypes bias sourcemonitoring attributions. Memory, 14, 148-160.

Mather, M., Henkel, L. A., \& Johnson, M. K. (1997). Evaluating characteristics of false memories: Remember/know judgments and memory characteristics questionnaire compared. Memory \& Cognition, 25, 826-837. 
Mather, M., \& Johnson, M. K. (2000). Choice-supportive source monitoring: Do our decisions seem better to us as we age? Psychology and Aging, 15, 596-606.

Mather, M., \& Johnson, M. K. (2003). Affective review and schema reliance in memory in older and younger adults. American Journal of Psychology, 116, 169-189.

Mather, M., Johnson, M. K., \& De Leonardis, D. M. (1999). Stereotype reliance in source monitoring: Age differences and neuropsychological test correlates. Cognitive Neuropsychology, 16, 437-458.

Mather, M., Knight, M., \& McCaffrey, M. (2005). The allure of the alignable: Younger and older adults' false memories of choice features. Journal of Experimental Psychology: General, $134,38-51$.

Mather, M., Shafir, E., \& Johnson, M. K. (2000). Misrememberance of options past: Source monitoring and choice. Psychological Science, 11, 132-138.

Mather, M., Shafir, E., \& Johnson, M. K. (2003). Remembering chosen and assigned options. Memory \& Cognition, 31, 422-434.

Neuschatz, J. S., Payne, D. G., Lampinen, J. M., \& Toglia, M. P. (2001). Assessing the effectiveness of warnings and the phenomenological characteristics of false memories. Memory, 9, 53-71.

Norman, K. A., \& Schacter, D. L. (1997). False recognition in younger and older adults: Exploring the characteristics of illusory memories. Memory \& Cognition, 25, 838-848.

Pizarro, D. A., Laney, C., Morris, E. K., \& Loftus, E. F. (2006). Ripple effects in memory: Judgments of moral blame can distort memory for events. Memory \& Cognition, 34, 550555. 
Roediger, H. L., \& McDermott, K. B. (1995). Creating false memories: Remembering words not presented in lists. Journal of Experimental Psychology: Learning, Memory, and Cognition, 21, 803-814.

Ross, M., McFarland, C., \& Fletcher, G. J. O. (1981). The effect of attitude on the recall of personal histories. Journal of Personality and Social Psychology, 37, 322-336.

Sanitioso, R., Kunda, Z., \& Fong, G. T. (1990). Motivated recruitment of autobiographical memories. Journal of Personality and Social Psychology, 59, 229-241.

Schaefer, A., \& Philippot, P. (2005). Selective effects of emotion on the phenomenal characteristics of autobiographical memories. Memory, 13, 148-160.

Sherman, J. W., \& Bessenoff, G. R. (1999). Stereotypes as source monitoring cues: On the interaction between episodic and semantic memory. Psychological Science, 10, 106-110.

Smith, J. A. (1994). Reconstructing selves: An analysis of discrepancies between women's contemporaneous and retrospective accounts of the transition to motherhood. British Journal of Psychology, 85, 371-392.

Spaniol, J., \& Bayen, U. J. (2002). When is schematic knowledge used in source monitoring? Journal of Experimental Psychology: Learning, Memory, and Cognition, 28, 631-651.

Talarico, J. M., LaBar, K. S., \& Rubin, D. C. (2004). Emotional intensity predicts autobiographical memory experience. Memory \& Cognition, 32, 1118-1132.

Zaragoza, M. S., \& Mitchell, K. J. (1996). Repeated exposure to suggestion and the creation of false memories. Psychological Science, 7, 294-300. 


\begin{abstract}
Author Note
Linda Henkel, Department of Psychology, Fairfield University, and Mara Mather, Department of Psychology, University of California, Santa Cruz.

This work was supported by grants from the National Science Foundation (0112284) and the National Institute on Aging (AG025340 and AG021443). Correspondence concerning this article can be addressed to Linda Henkel, Department of Psychology, Fairfield University, Fairfield, CT 06824. Electronic mail may be sent to lhenkel@mail.fairfield.edu or to mather@ucsc.edu.
\end{abstract}




\section{Footnotes}

${ }^{1}$ The asymmetry score calculation performed for each person on each choice scenario is outlined below (for more detail and discussion, see Mather et al., 2000). The extent to which the person's memory attributions favored an option (call it option A) was computed by subtracting the proportion of attributions favoring the competing option (option B) from those favoring A (positive features attributed to Option A and negative features attributed to Option B both favor Option A). The resulting sums were then converted to $z$-scores such that the mean value across all participants was zero. Relative to the mean, a positive value indicates that the person's attributions favor option $\mathrm{A}$, whereas a negative value indicates favoring of option $\mathrm{B}$. This score was left intact for those who chose option A (thus capturing the extent to which they favored their chosen option), whereas for people who chose option B, the score was multiplied by -1 . This formula was repeated for separate subcomponents, such as just the features correctly attributed and just the new features incorrectly attributed to an option.

2 In Experiment 1, the ratios of people choosing one option over another for each of the five scenarios ranged from a relatively even split or a slight favoring of one option $(50: 50,61: 39)$ to a stronger preference for one of the options $(67: 33,78: 22,81: 19)$. Similarly in Experiment 2, there were scenarios where the preference was not very pronounced $(51: 49,54: 46,56: 44)$ and scenarios with a more strongly preferred option $(71: 39,74: 26)$, one of which was the car scenario which was not included with the data analyses because it was a filler scenario. 
Table 1

Car Scenario Choice Options (Valence of Features was not Indicated for Participants)

Option 1: Red car $(5+, 5-)$

- Hard to find service outlets

- Has a dent from a previous accident

+ Seats are very comfortable

+ Good handling on turns

- High mileage on odometer

- Makes an unidentified rattling sound

+ Prestigious model

+ Air conditioning included

- Doesn't do well in bad weather

+ Stereo included

Option 2: Black car $(5+, 5-)$

- No warranty

- Some rust on exterior

+ High resale value

+ Has airbags

- Needs a few repairs

- Not much trunk space

+ Powerful engine

+ Previous owner took good care of car

- Not fuel efficient

+ Has a sun roof 
Table 2

Hits, False Alarms, and Source Identification Accuracy for Positive and Negative Features in Experiment 1 (SEs given in parentheses)

Proportion Attributed

Feature Valence

Positive $\quad$ Negative $\quad M_{\text {diff }}(95 \% \mathrm{CI})$

Correctly Remembered Which Option Was Chosen $(n=80)$

Hits to Studied Items $\quad .82(.01) \quad .88(.01) \quad-.06( \pm .02)$

False Alarms to New Items $\quad .43(.02) \quad .28(.02) \quad .15( \pm .03)$

Source Identification Accuracy $\quad .60(.01) \quad$.68 $\quad .01) \quad-.08( \pm .03)$

Misremembered Which Option Was Chosen $(n=50)$

Hits to Studied Items $\quad .83(.02) \quad .90(.02) \quad$. $\quad .08( \pm .04)$

False Alarms to New Items $\quad .47(.05) \quad .32(.04) \quad .14( \pm .10)$

Source Identification Accuracy $\quad .55(.03) \quad .56(.02) \quad .01( \pm .06)$ 
Table 3

Choice-supportive Asymmetry Scores When Participants Correctly Believe They Chose the Chosen Option Versus When They Incorrectly Believe They Chose the Rejected Option in Experiments 1 and 2

\begin{tabular}{lll} 
Old Features & Old Features & New Features \\
Correctly & Incorrectly & Incorrectly \\
Attributed & Attributed & Attributed \\
\hline
\end{tabular}

Experiment 1

$\begin{array}{llll}\text { Correct Belief } & .26(.08) & .18(.08) & .25(.09) \\ \text { Incorrect Belief } & .46(.14) & .50(.14) & .41(.14)\end{array}$

Experiment 2

$\begin{array}{llll}\text { Correct Belief } & .41(.08) & .40(.09) & .36(.10) \\ \text { Incorrect Belief } & .41(.11) & .47(.11) & .42(.13)\end{array}$

Note: Positive scores indicate a bias in favor of the option believed to be the chosen option. Standard errors are in parentheses. 
Table 4

Hits, False Alarms, and Source Identification Accuracy for Positive and Negative Features in Experiment 2 (SEs given in parentheses)

Proportion Attributed

Feature Valence

Positive $\quad$ Negative $\quad M_{\text {diff }}(95 \% \mathrm{CI})$

Correct-Reminder Scenarios

Hits to Old Items

False Alarms to New Items

Source Identification Accuracy

Misleading-Reminder Scenarios

Hits to Old Items

False Alarms to New Items

Source Identification Accuracy
$.79(.02)$

$.37(.04)$

$.60(.02)$

$.61(.02) \quad-.01( \pm .04)$
$.61(.02) \quad-.01( \pm .04)$

$-.03( \pm .02)$

$.30(.03) \quad .08( \pm .08)$
$.79(.02) \quad .80(.02) \quad-.01( \pm .03)$

$.38(.03) \quad .27(.03) \quad .11( \pm .07)$

$.47(.02) \quad .48(.02) \quad-.01( \pm .04)$ 


\section{Figure Captions}

Figure 1. Proportion of features attributed to the option believed to be chosen or rejected as a function of valence of feature in Experiment 1 (error bars represent SE). The top panel represents attributions for studied features, and the bottom panel represents attributions for new features.

Figure 2. Proportion of features attributed to the option believed to be chosen or rejected as a function of valence of feature in Experiment 2 (error bars represent SE). The top panel represents attributions for studied features, and the bottom panel represents attributions for new features.

Figure 3. Memorial characteristic ratings for features from scenarios in Experiment 2 for which participants' beliefs about which options they chose were correct (correct reminder compared with those for which their beliefs were wrong (misleading reminder). Black bars indicate ratings for features attributed in a choice-supportive (CS) fashion and white bars indicate ratings for features attributed in a non choice-supportive (NCS) fashion. Error bars indicate the standard error of the mean. 


\section{ATTRIBUTIONS FOR STUDIED FEATURES}

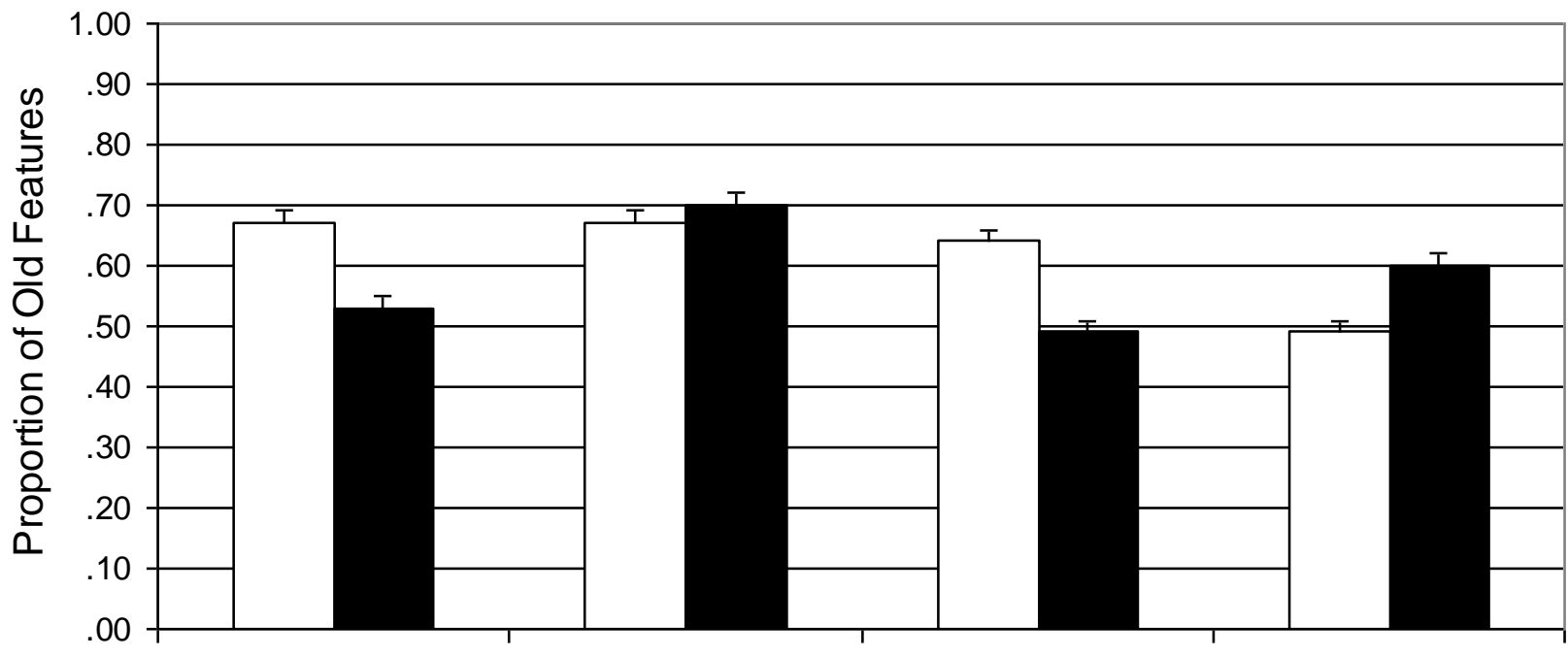

Positive Features Negative Features Positive Features Negative Features

Correctly Remembered Choices

Misremembered Choices

\section{ATTRIBUTIONS FOR NEW FEATURES}

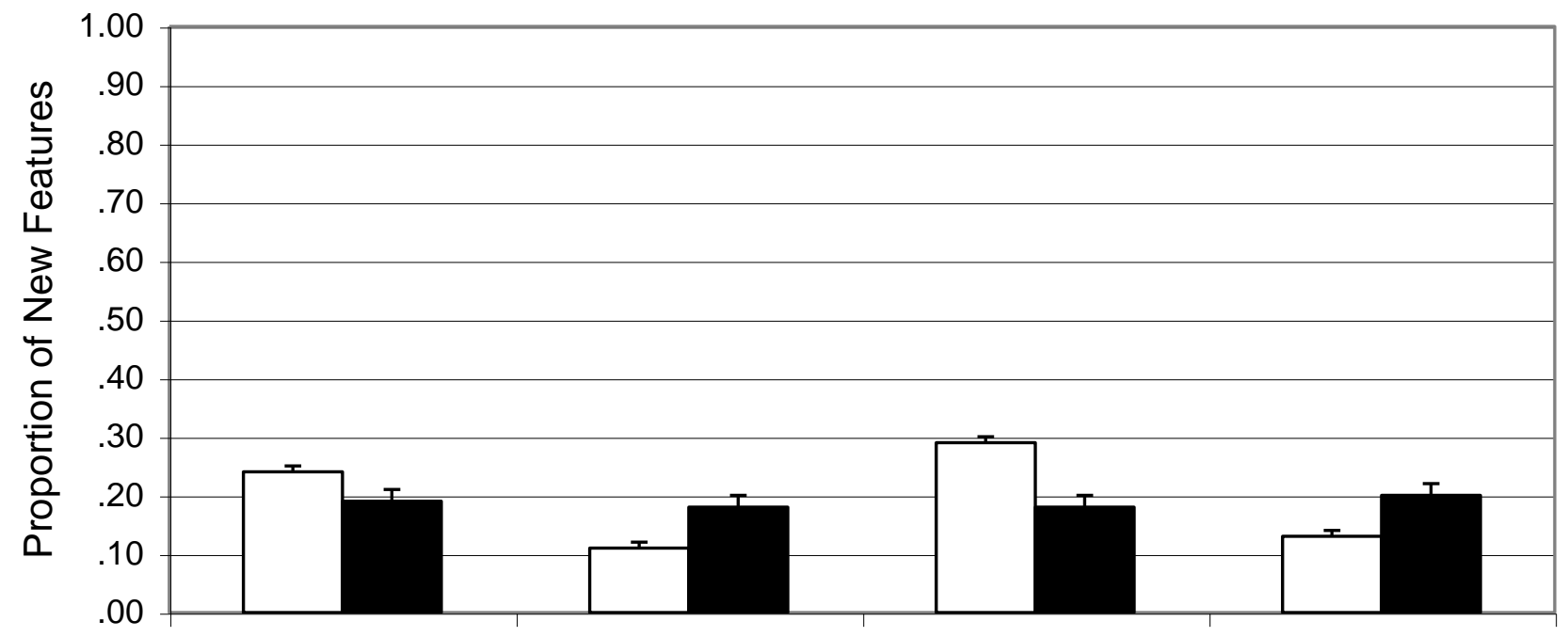

Positive Features Negative Features Positive Features Negative Features

Correctly Remembered Choices

Misremembered Choices

$\square$ Attributed to Option Remembered as Chosen Attributed to Option Remembered as Rejected 


\section{ATTRIBUTIONS FOR STUDIED FEATURES}

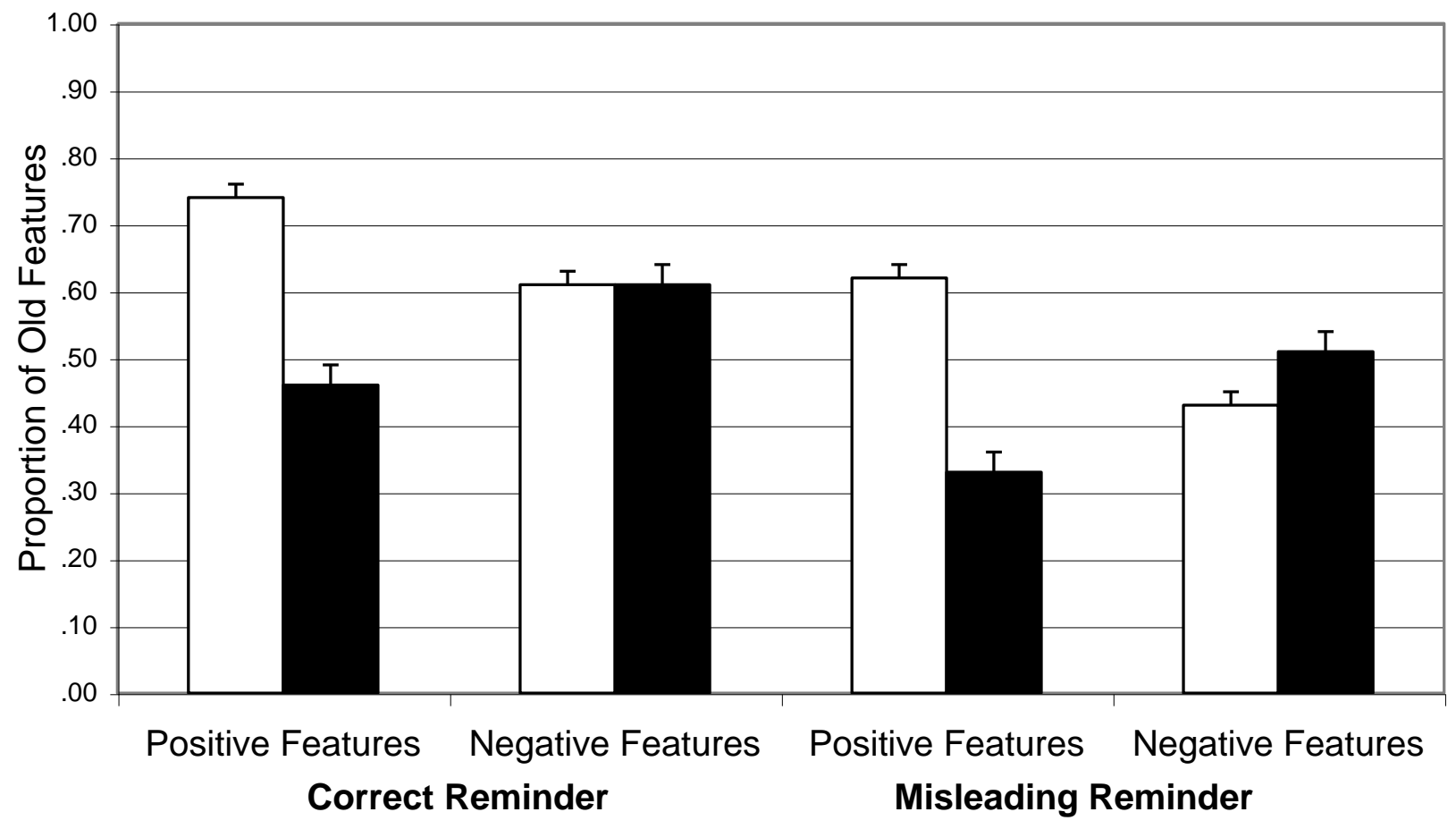

ATTRIBUTIONS FOR NEW FEATURES

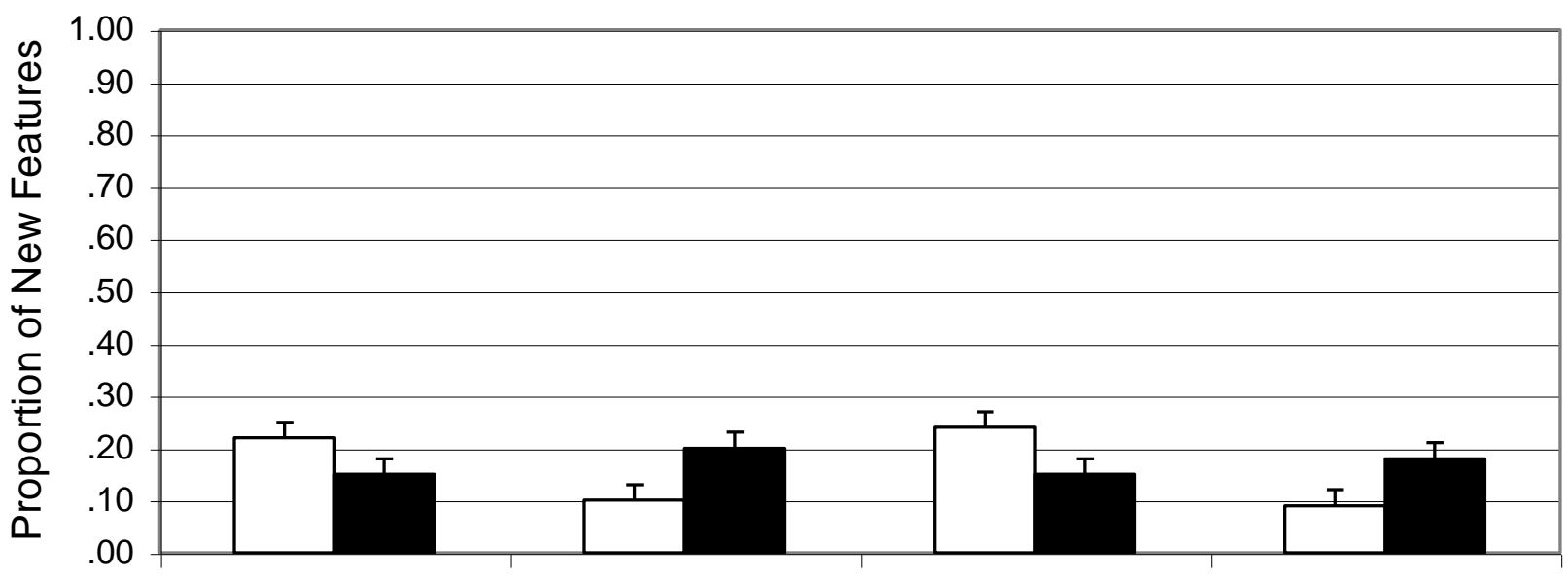

Positive Features Negative Features Positive Features Negative Features Correct Reminder 

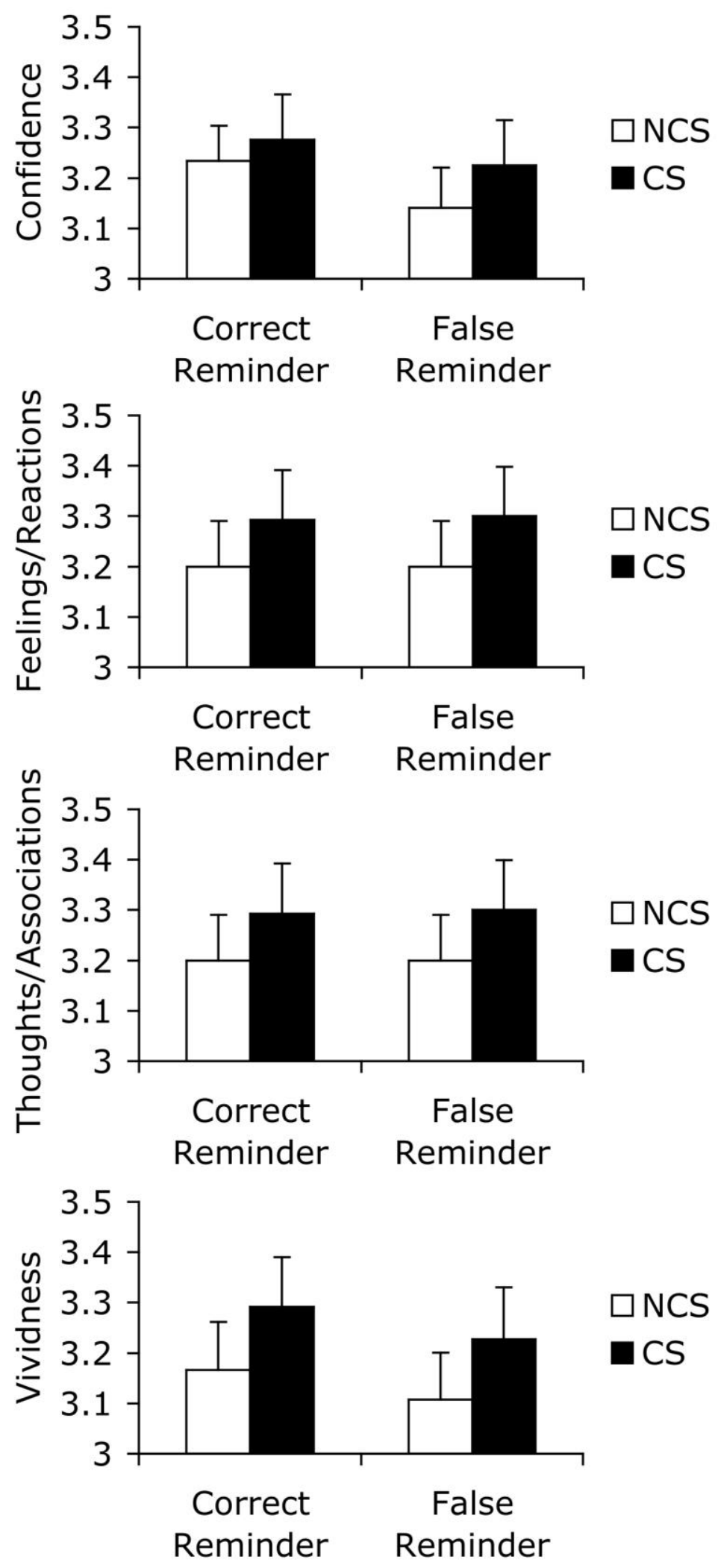